\title{
Concomitant aortic valve repair with continuous-flow left ventricular assist devices: Results and implications
}

\author{
Shinichi Fukuhara, MD, ${ }^{\text {a }}$ Koji Takeda, MD, PhD, ${ }^{\text {a }}$ Codruta Chiuzan, $\mathrm{PhD},{ }^{\mathrm{c}}$ Jiho Han, BS, \\ Antonio R. Polanco, MD, ${ }^{\mathrm{a}}$ Melana Yuzefpolskaya, MD, ${ }^{\mathrm{b}}$ Donna M. Mancini, MD, ${ }^{\mathrm{b}}$ \\ Paolo C. Colombo, MD, ${ }^{\mathrm{b}}$ Veli K. Topkara, MD, ${ }^{\mathrm{b}}$ Paul A. Kurlansky, MD, ${ }^{\mathrm{a}}$ Hiroo Takayama, MD, PhD, ${ }^{\mathrm{a}}$ and \\ Yoshifumi Naka, MD, PhD
}

\begin{abstract}
Objectives: Aortic insufficiency (AI) after continuous-flow left ventricular assist device implantation can affect patient outcomes. Central aortic valve closure (CAVC) is a strategy commonly practiced; however, its efficacy has not been extensively investigated.
\end{abstract}

Methods: From March 2004 to May 2014, a total of 340 patients received a continuous-flow left ventricular assist device $(89 ; 26.2 \%)$ as destination therapy (DT). Outcomes were compared between patients with CAVC $(n=57[16.8 \%]$; group A) versus without repair $(\mathrm{n}=283$ [83.2\%]; group $\mathrm{B})$.

Results: Patients in group A were older, were more likely to be having DT, had a greater cardiopulmonary bypass and aortic crossclamp time, and more often received intraoperative transfusions than did patients in group $B$. Twenty-three $(40.4 \%)$ patients in group A had significant pre-existing AI, defined as $>$ mild AI, whereas none did in group B. Kaplan-Meier analysis revealed that freedom from significant $\mathrm{AI}$ was $66.7 \%$ and $59.9 \%$ at 2 years $(P=.77)$ in groups $\mathrm{A}$ and $\mathrm{B}$, respectively. In the DT cohort, freedom from significant AI was 78.1\% and $41.8 \%$ at 2 years $(P=.077)$. A generalized mixed-effects model demonstrated a $57 \%$ and $69 \%$ decrease in the odds of significant AI progression among repaired patients in the entire and DT cohort, respectively, after adjusting for time effect and degree of baseline pre-existing AI.

Conclusions: Despite pre-existing AI, the prevalence of significant AI in patients with CAVC was comparable to the AI in those without pre-existing AI/CAVC. The efficacy of this technique was more evident in DT patients. Thus, CAVC may be an effective and durable strategy, especially in patients who require lengthy device support. (J Thorac Cardiovasc Surg 2016;151:201-10)

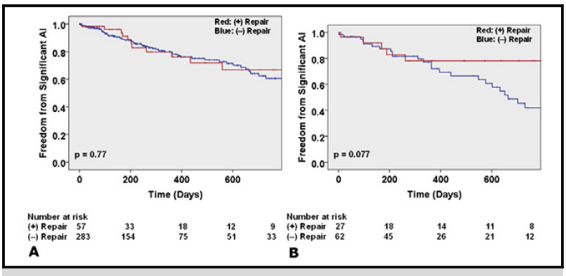

Freedom from significant aortic insufficiency: patients with, versus without, repair, for (A) entire cohort and (B) destination therapy subgroup.

\section{Central Message}

Concomitant aortic valve repair may be an effective strategy in addressing pre-existing aortic insufficiency for device-supported patients.

\section{Perspective}

The present study is the largest series describing concomitant aortic valve repair in patients undergoing continuous-flow left ventricular assist device placement. Concomitant central aortic valve closure may be an effective and durable strategy in addressing pre-existing aortic insufficiency for device-supported patients, especially those expected to require multiyear device support, such as those in destination therapy.

See Editorial Commentary page 211

See Editorials page 10 and 13 .

\footnotetext{
From the Divisions of a Cardiac, Thoracic and Vascular Surgery and ${ }^{\mathrm{b}}$ Cardiology, Columbia University Medical Center, and ${ }^{\mathrm{c}}$ Department of Biostatistics, Columbia University Mailman School of Public Health, New York, NY.

Read at the 95th Annual Meeting of The American Association for Thoracic Surgery, Seattle, Washington, April 25-29, 2015.

Received for publication March 6, 2015; revisions received Sept 2, 2015; accepted for publication Sept 26, 2015.

Address for reprints: Yoshifumi Naka, MD, PhD, Division of Cardiac, Thoracic and Vascular Surgery, Columbia University Medical Center, 177 Fort Washington Ave, New York, NY 10032 (E-mail: yn33@cumc.columbia.edu). $0022-5223 / \$ 36.00$

Copyright (c) 2016 by The American Association for Thoracic Surgery http://dx.doi.org/10.1016/j.jtcvs.2015.09.128
} 


\section{Abbreviations and Acronyms \\ AI $=$ aortic insufficiency \\ $\mathrm{AV} \quad=$ aortic valve \\ CAVC $=$ central aortic valve closure \\ CF-LVAD = continuous-flow left ventricular assist device \\ DT $=$ destination therapy \\ INTERMACS $=$ Interagency Registry for \\ Mechanically Assisted Circulatory Support}

earlier-generation pulsatile LVADs, they have rapidly become integral to the treatment of patients who have advanced heart failure..$^{1-3}$ The recipients of CF-LVADs are more likely than recipients of pulsatile LVADs to develop worsening aortic insufficiency (AI). ${ }^{4}$ Therefore, progressive AI, either de novo or developing from pre-existing $\mathrm{AI}$, and its impact on clinical outcomes, has received increasing attention. ${ }^{5,6}$ With an increasing number of patients being supported with longer-term usage of CF-LVADs, investigation of the optimal management of AI progression has become compelling.

Patients with pre-existing AI have undergone aortic valve (AV) surgery concomitantly with or or after undergoing implantation, including AV closure, AV repair with varied techniques, ${ }^{7,8}$ AV replacement with a bioprosthesis, ${ }^{9}$ and more recently, percutaneous device closure. ${ }^{10}$ However, data are scarce regarding the applicability, efficacy, and durability of each strategy, mainly because of the small sample size and limited follow-up periods. Central aortic valve closure (CAVC), first described by Park and colleagues,${ }^{11}$ has been a commonly practiced strategy to eliminate central regurgitation while allowing opening of the $\mathrm{AV}$ through the lateral aspect of the valve leaflets. ${ }^{12}$ Previous studies describing this technique were performed on short- and/or mid-term data only, from small patient cohorts. ${ }^{12,13}$ Therefore, the optimal management approach for pre-existing AI remains to be determined. We have reviewed our experience with CF-LVAD implantation, with and without concomitant CAVC, to assess its clinical efficacy, which is defined as a reduction of native $\mathrm{AI}$ to trace or none at the time of repair, and a decreased prevalence of greater-than-mild AI during CF-LVAD support.

\section{METHODS}

\section{Patients and Study Design}

The Columbia University Institutional Review Board approved all aspects of the present study, and individual patient consent was waived. We retrospectively reviewed 360 patients who underwent CF-LVAD implantation between March 2004 and May 2014. Of these, 4 patients who underwent concomitant $\mathrm{AV}$ replacement were excluded: 3 with concomitant $\mathrm{AV}$ closure with a Dacron or bovine pericardial patch; 1 with concomitant AV repair using a different technique; and 9 who had previously undergone $\mathrm{AV}$ procedures ( 8 replacements; 1 repair). Additionally, 3 individuals were excluded who received the Jarvik 2000 (Jarvik Heart, New York, NY) via left thoracotomy. A total of 340 patients were included in this analysis (Figure E1). Subjects were grouped into group A $(n=57[16.8 \%]$ with concomitant CAVC) and group B (283 [83.2\%] without CAVC).

Abstracted data included the following: patient demographics, clinical and treatment variables, cardiopulmonary bypass and aortic crossclamp time, blood product use, dosage of vasoactive drugs at the time of device implantation, perioperative and follow-up echocardiographic variables, freedom from $\mathrm{AI}$ occurrence, number of adverse events, and survival in each group. Follow-up was complete as of October 1, 2014. The completion rate was $96.5 \%$.

\section{Devices Implanted}

The types of CF-LVADs implanted are as follows: the HeartMate II (Thoratec Corporation, Pleasanton, Calif) $(\mathrm{n}=281 ; 82.6 \%)$; the HeartWare Ventricular Assist System (HeartWare International, Inc, Framingham, Mass) $(\mathrm{n}=36 ; 10.6 \%)$; the VentrAssist (Ventracor Ltd, Chatswood, New South Wales, Australia) $(\mathrm{n}=9 ; 2.6 \%)$; the DuraHeart (Terumo Heart, Inc, Ann Arbor, Mich) ( $\mathrm{n}=8 ; 2.4 \%)$; and the MicroMed DeBakey (MicroMed Technology, Inc, Houston, Tex $)(\mathrm{n}=6 ; 1.8 \%)$.

\section{Assessment and Follow-Up of Aortic Insufficiency}

Details of our protocol for echocardiographic evaluation have been reported elsewhere. ${ }^{14}$ In brief, serial transthoracic echocardiography and intraoperative transesophageal echocardiography evaluations were performed in all patients. The presence of AI was determined at baseline, prior to device implantation, and until the time of the last follow-up or censoring event, as clinically indicated, usually at 30 days, 3 months, 6 months, and annually thereafter. Aortic insufficiency was evaluated visually in the parasternal short- and long-axis views by transthoracic echocardiography and graded on an interval scale, based on AI jet width, according to the recommendations of the American Society of Echocardiography, as 1 of the following: none, trace, mild, mild to moderate, moderate, moderate to severe, or severe. ${ }^{15}$

\section{Indications and Operative Technique of Central Aortic Valve Closure}

All patients with pre-existing AI graded as $>$ mild, or DT patients with mild AI, received a concurrent CAVC. In addition, 2 DT patients with trace AI underwent repair at the discretion of the surgeon. Cardiopulmonary bypass was established with the standard aorto-atrial cannulation, unless bicaval cannulation was needed for other procedures. The patient was cooled to $32^{\circ} \mathrm{C}$, the aorta was crossclamped, and cardioplegia was administered through the aortic root. A transverse aortotomy was made to approach the AV. The repair was performed by approximating the center of the leaflets with a 4-0 pledgeted polypropylene suture. Typically, the pledget was made of CorMatrix ECM (CorMatrix Cardiovascular, Inc, Atlanta, Ga) or woven polyester from the outflow graft of the device. After completion of the CAVC and aortotomy closure, the aortic crossclamp was removed. The rest of the procedure was conducted in the usual fashion with a beating heart. ${ }^{16}$

\section{Statistical Analysis}

Continuous variables were expressed as mean \pm 1 SD. Categoric variables were presented as proportions and absolute numbers. Differences were detected using $\chi^{2}$ analysis or the Fisher exact test for categoric variables, and the Mann-Whitney $U$ or Student $t$ test for continuous variables. The cumulative probability of on-device survival and significant AI (defined as >mild) were each estimated using the Kaplan-Meier method, and compared with those from the log-rank test. Patients were censored for transplantation and device explantation for myocardial recovery for the former; for the latter, they were censored for transplantation, death, and device explantation. The time spent on device support was calculated from the date of the first device implantation. 
For longitudinal data analysis, a mixed-effects logistic regression model with a logit link was employed to assess the association between CAVC and AI progression. This type of model is useful for incomplete longitudinal dichotomous outcomes, for which data are assumed to be missing at random. ${ }^{17}$ The final models included fixed effects for treatment group, time (continuous scale), and baseline AI degree (ordinal scale), as well as a random effect to account for within-subject variation. To satisfy the linearity assumption, a $\log$ transformation was applied to the time variable. In addition, a time $\times$ group interaction was tested and found to be statistically nonsignificant. Preimplantation clinical parameters were analyzed to determine predictors for post-device implantation AI progression, using Cox proportional hazards models. For multivariable analysis, variables with a $P$ value $\leq .25$ on univariable analysis were included in a final model.

Results are presented as hazard ratios, with corresponding $95 \%$ confidence intervals. All $P$ values were results of 2 -tailed tests. The statistical analysis was performed using SPSS version 22.0 (SPSS, Inc, Chicago, Ill) and SAS version 9.4 (SAS Institute, Inc, Cary, NC).

\section{RESULTS}

\section{Patient Baseline Characteristics}

Patient demographic and clinical variables are shown in Table 1 . The mean age was 56.6 years; $66(19.4 \%)$ were women, and $89(26.2 \%)$ received a device as DT. The mean follow-up period and device support duration were $2.3 \pm 2.0$ years, and $423.6 \pm 453.2$ days, respectively. Patients in group A were older (65.1 vs $54.9 ; P<.001)$, were more likely to be DT ( 47.4 vs $21.9 \% ; P<.001)$, and had a higher blood urea nitrogen level $(41.9 \pm 21.6$ vs $33.7 \pm 19.7 ; P=.005$ ) and a higher HeartMateII risk score $^{18}(2.09$ vs $1.50 ; P<.001)$ than did patients in group B. Otherwise, the groups displayed similar clinical characteristics.

The baseline echocardiographic variables are further displayed in Table 2. Twenty-three (40.4\%) patients in group A had significant pre-existing AI, defined as $>$ mild AI, comprising $9(15.8 \%)$ mild to moderate, $11(19.3 \%)$ moderate, $1(1.8 \%)$ moderate to severe, and $2(3.5 \%)$ severe; none of the patients in group B were in this category $(P<.001)$. Moderate or greater mitral, and/or tricuspid, regurgitation were common. The aortic root diameter was larger in group A.

\section{Intraoperative Data}

The intraoperative data show that subjects in group A had a greater cardiopulmonary bypass time $(112.3 \pm 44.2 \mathrm{vs}$ $90.7 \pm 48.1$ minutes; $P<.001)$ and aortic crossclamp time $(23.3 \pm 9.9$ vs $1.6 \pm 8.1$ minutes; $P=.002)$, and more often received transfusions than did those in group B (Table 3). In addition, the dosage of vasoactive drugs, as represented by the vasoactive-inotropic score, ${ }^{19}$ was higher in the former group. In addition, patients in group A received a concomitant mitral repair more often than did those in group B.

\section{Adverse Events and Survival}

Early postoperative outcomes and overall adverse event results showed that non-device-related infections were more frequent in group A (Tables 4 and 5). No difference was found regarding the occurrence of other adverse events. The estimated on-device survival in groups $\mathrm{A}$ and $\mathrm{B}$, respectively, were $84.6 \% \pm 5.1 \%$ and $83.4 \% \pm 2.6 \%$ at 1 year $(P=.98)$, and $65.2 \% \pm 9.4 \%$ and $77.8 \% \pm 3.4 \%$ at 2 years $(P=.41)$ (Figure E2 and Table E2). A total of $157(46.2 \%)$ patients were bridged to transplantation with a mean support duration of 322 days. At the latest follow-up, $131(38.5 \%)$ patients were alive after the transplant; $115(33.8 \%)$ remained on device support; and $90(26.5 \%)$ had died. In addition, no difference was observed for posttransplantation survival at 3 years $(84.2 \%$ vs $86.2 \%, P=.81)$ among those bridged to transplant.

\section{Post-Device Implantation Aortic Insufficiency Progression}

For the entire cohort, freedom from significant AI was $66.7 \% \pm 8.9 \%$ and $59.9 \% \pm 5.1 \%$ at 2 years $(P=.77)$, in groups $\mathrm{A}$ and $\mathrm{B}$, respectively (Figure $1, A$ ); for the DT cohort, the percentages were $78.1 \% \pm 8.7 \%$ and $41.8 \% \pm 8.3 \%(P=.077)$ (Figure $1, B)$. No subjects in group A developed significant AI beyond 1 year after device placement. In group B, $41.7 \%$ had significant AI, in the period between 1 and 2 years after device implantation. Furthermore, the results of an additional mixed-effects model, used to address longitudinal data, are summarized in Table 6.

Both time and baseline AI degree had a significant association with AI progression. For every unit increase in baseline AI degree, the odds of developing a significant AI were $34 \%$ higher (odds ratio $=1.34$, with $95 \%$ confidence interval 1.06-1.70). After adjustment for time and baseline AI degree, the decrease in the odds that a patient in group A would develop a significant AI decreased by $57 \%$ (odds ratio $=0.43$, with $95 \%$ confidence interval $0.20-0.93 ; P=.032$ ) (Table 6). The results were similar for the DT cohort, with a $69 \%$ decrease in the odds of significant AI progression (odds ratio $=0.31$, with $95 \%$ confidence interval $0.12-0.79$; $P=.015)$ (Table 6).

Another subgroup analysis concerning AI progression among patients with mild pre-existing $\mathrm{AI}$ was performed. A total of 44 patients (32 in group A and 12 in group B) had mild AI at baseline. The comparison between groups revealed no difference with respect to progression to $\geq$ moderate $\mathrm{AI}$ ( 4 of $32[12.5 \%$ ] in group A vs 1 of $12(8.3 \%)$ in group $\mathrm{B} ; P=.70)$ over a median device support period of 207 days (interquartile range: 88 to 511 days). 
TABLE 1. Patient demographics and clinical characteristics before device implantation

\begin{tabular}{|c|c|c|c|c|}
\hline Variable & All & With repair & Without repair & $P$ value \\
\hline Number of patients & 340 & $57(16.8)$ & $283(83.2)$ & \\
\hline Gender, female & $66(19.4)$ & $6(10.5)$ & $60(21.2)$ & .063 \\
\hline Age (y) & $56.6 \pm 13.9$ & $65.1 \pm 8.3$ & $54.9 \pm 14.1$ & $<.001 *$ \\
\hline Aged $\geq 65 \mathrm{y}$ & $114(33.5)$ & $31(54.4)$ & $83(29.3)$ & $<.001 *$ \\
\hline Race/ethnicity, nonwhite & $188(55.3)$ & $30(52.6)$ & $158(55.8)$ & .66 \\
\hline Body mass index $\left(\mathrm{kg} / \mathrm{m}^{2}\right)$ & $26.3 \pm 5.7$ & $25.9 \pm 5.5$ & $26.4 \pm 5.7$ & .53 \\
\hline Etiology & & & & .26 \\
\hline Ischemic cardiomyopathy & $148(43.5)$ & $30(52.6)$ & $118(41.7)$ & \\
\hline Dilated cardiomyopathy & $171(50.3)$ & $23(40.4)$ & $148(52.3)$ & \\
\hline Other & $21(6.4)$ & $4(7.0)$ & $17(6.0)$ & \\
\hline Device intent & & & & $<.001^{*}$ \\
\hline Bridge-to-transplant & $251(73.8)$ & $30(52.6)$ & $221(78.1)$ & \\
\hline Destination therapy & $89(26.2)$ & $27(47.4)$ & $62(21.9)$ & \\
\hline \multicolumn{5}{|l|}{ Medical comorbidities } \\
\hline Hypertension & $168(49.4)$ & $32(56.1)$ & $136(48.1)$ & .27 \\
\hline Dyslipidemia & $124(36.5)$ & $26(45.6)$ & $98(34.6)$ & .12 \\
\hline Coronary artery disease & $149(43.8)$ & $28(49.1)$ & $121(42.8)$ & .38 \\
\hline Diabetes & $116(34.1)$ & $18(31.6)$ & $98(34.6)$ & .66 \\
\hline COPD & $33(9.7)$ & $10(17.5)$ & $23(8.1)$ & $.028 *$ \\
\hline Prior CVA & $41(12.1)$ & $9(15.8)$ & $32(11.3)$ & .34 \\
\hline Previous malignancy & $37(10.9)$ & $7(12.2)$ & $30(10.6)$ & .71 \\
\hline Drug abuse & $18(5.3)$ & $5(8.8)$ & $13(4.6)$ & .20 \\
\hline Smoking & $113(33.3)$ & $23(40.4)$ & $90(31.8)$ & .21 \\
\hline \multicolumn{5}{|l|}{ Previous cardiac surgery } \\
\hline CABG & $70(20.6)$ & $17(29.8)$ & $53(18.7)$ & .059 \\
\hline Mitral & $24(7.1)$ & $9(15.8)$ & $15(5.3)$ & $.005^{*}$ \\
\hline Tricuspid & $5(1.5)$ & $1(1.8)$ & $4(1.4)$ & .85 \\
\hline VAD/ECMO & $35(10.3)$ & $6(14.0)$ & $29(10.2)$ & .95 \\
\hline $\mathrm{AICD}$ & $270(79.4)$ & $42(73.7)$ & $228(80.6)$ & .24 \\
\hline Other & $11(3.2)$ & $1(1.8)$ & $10(3.5)$ & .49 \\
\hline IABP & $88(25.9)$ & $17(29.8)$ & $71(25.1)$ & .46 \\
\hline Inotrope & $271(79.7)$ & $39(68.4)$ & $232(82.0)$ & $.02 *$ \\
\hline Vasopressor & $32(9.4)$ & $5(8.8)$ & $27(9.5)$ & .86 \\
\hline Mechanical ventilation & $16(4.7)$ & $5(8.8)$ & $11(3.9)$ & .11 \\
\hline \multicolumn{5}{|l|}{ Laboratory values } \\
\hline BUN (mg/dL) & $35.1 \pm 20.3$ & $41.9 \pm 21.6$ & $33.7 \pm 19.7$ & $.005^{*}$ \\
\hline Creatinine (mg/dL) & $1.51 \pm 0.77$ & $1.60 \pm 0.65$ & $1.49 \pm 0.79$ & .35 \\
\hline Total protein $(\mathrm{g} / \mathrm{dL})$ & $6.5 \pm 1.2$ & $6.4 \pm 0.8$ & $6.5 \pm 1.3$ & .79 \\
\hline Albumin $(\mathrm{g} / \mathrm{dL})$ & $3.6 \pm 0.5$ & $3.5 \pm 0.6$ & $3.6 \pm 0.5$ & .14 \\
\hline Total bilirubin $(\mathrm{mg} / \mathrm{dL})$ & $1.5 \pm 1.1$ & $1.4 \pm 1.1$ & $1.5 \pm 1.1$ & .59 \\
\hline Direct bilirubin (mg/dL) & $0.5 \pm 0.6$ & $0.5 \pm 0.5$ & $0.5 \pm 0.6$ & .99 \\
\hline $\mathrm{AST}(\mathrm{U} / \mathrm{L})$ & $45.7 \pm 92.1$ & $64.9 \pm 169.8$ & $41.8 \pm 66.0$ & .32 \\
\hline ALT (U/L) & $57.4 \pm 153.0$ & $53.0 \pm 130.9$ & $58.3 \pm 157.3$ & .81 \\
\hline $\operatorname{ALP}(\mathrm{U} / \mathrm{L})$ & $98.1 \pm 52.1$ & $99.7 \pm 48.1$ & $97.7 \pm 52.9$ & .80 \\
\hline PT/INR & $1.36 \pm 0.40$ & $1.46 \pm 0.54$ & $1.34 \pm 0.36$ & .14 \\
\hline \multicolumn{5}{|l|}{ Hemodynamic parameters } \\
\hline Systolic blood pressure (mm Hg) & $102.0 \pm 13.4$ & $101.0 \pm 11.1$ & $102.2 \pm 13.7$ & .59 \\
\hline Central venous pressure $(\mathrm{mm} \mathrm{Hg})$ & $11.0 \pm 5.5$ & $9.7 \pm 4.5$ & $11.2 \pm 5.6$ & .068 \\
\hline Mean pulmonary pressure $(\mathrm{mm} \mathrm{Hg})$ & $34.8 \pm 9.5$ & $35.6 \pm 9.4$ & $34.6 \pm 9.5$ & .49 \\
\hline Wedge pressure $(\mathrm{mm} \mathrm{Hg})$ & $23.7 \pm 7.9$ & $24.5 \pm 7.5$ & $23.6 \pm 8.0$ & .50 \\
\hline Cardiac index $\left(\mathrm{L} / \mathrm{min} / \mathrm{m}^{2}\right)$ & $1.7 \pm 0.5$ & $1.7 \pm 0.4$ & $1.7 \pm 0.5$ & .55 \\
\hline PVR (Wood units) & $3.9 \pm 2.7$ & $3.8 \pm 2.0$ & $4.0 \pm 2.8$ & .68 \\
\hline INTERMACS profiles & & & & .26 \\
\hline $1-2$ & $253(74.4)$ & $39(68.4)$ & $214(75.6)$ & \\
\hline $3-4$ & $87(25.6)$ & $18(31.6)$ & $69(24.4)$ & \\
\hline
\end{tabular}


TABLE 1. Continued

\begin{tabular}{lccr}
\hline \multicolumn{1}{c}{ Variable } & All & With repair & Without repair \\
\hline HeartMate II risk score & $1.61 \pm 0.92$ & $2.09 \pm 0.80$ & $1.50 \pm 0.93$ \\
HeartMate II risk score profiles & & & $<.001^{*}$ \\
High & $44(12.9)$ & $14(24.6)$ & $30(10.6)$ \\
Medium & $122(35.9)$ & $27(47.4)$ & $95(33.6)$ \\
Low & $174(51.2)$ & $16(28.1)$ & $158(55.8)$ \\
\hline
\end{tabular}

Values are n $(\%)$, or mean $\pm \mathrm{SD}$, unless otherwise indicated. $C O P D$, Chronic obstructive pulmonary disorder; $C V A$, cerebrovascular accident; $C A B G$, coronary artery bypass grafting; $V A D$, ventricular assist device; $E C M O$, extracorporeal membrane oxygenation; $A I C D$, automated implantable cardioverter-defibrillator; $I A B P$, intra-aortic balloon pump; $B U N$, blood urea nitrogen; $A S T$, aspartate aminotransferase; $A L T$, alanine aminotransferase; $A L P$, alkaline phosphatase; $P T$, prothrombin time; $I N R$, International Normalized Ratio; $P V R$, pulmonary vascular resistance; INTERMACS, Interagency Registry for Mechanically Assisted Circulatory Support. $* P<.05$.

During the follow-up period, $5(8.8 \%)$ patients in group A, and $27(9.5 \%)$ in group B, developed $\geq$ moderate AI. Of these, $1(20.0 \%)$ in group A, and $10(37.0 \%)$ in group B required surgical interventions to address clinically significant, severe AI refractory to medical management (Table 7$)$. One $(1.7 \%)$ in group $\mathrm{A}$, and $3(1.1 \%)$ in group $\mathrm{B}$, developed heart failure that required an urgent United Network for Organ Sharing status upgrade and resultant transplantation. Five $(1.8 \%)$ underwent CAVC, and $2(0.8 \%)$ had $\mathrm{AV}$ closure and replacement, respectively. One patient $(0.4 \%)$ underwent percutaneous AV closure with an AMPLATZER (St Jude Medical, Inc, Minnetonka, Minn) and required AV replacement after the initial treatment failure. The 5 patients who additionally received CAVC remain well, and free of AI progression, with a mean follow-up period of 501 days, except for a patient who died from sepsis on the 21st postoperative day.

TABLE 2. Baseline echocardiographic characteristics before device implantation

\begin{tabular}{|c|c|c|c|c|}
\hline Variable & All & With repair & $\begin{array}{c}\text { Without } \\
\text { repair }\end{array}$ & $\begin{array}{c}P \\
\text { value }\end{array}$ \\
\hline LVEF & $15.2 \pm 6.2$ & $18.5 \pm 8.6$ & $14.5 \pm 5.4$ & $.004 *$ \\
\hline Degree of AI & & & & $<.001 *$ \\
\hline None & $166(48.8)$ & 0 & $156(58.7)$ & \\
\hline Trace & $107(31.5)$ & $2(3.5)$ & $105(37.1)$ & \\
\hline Mild & 44 (12.9) & $32(56.1)$ & $12(4.2)$ & \\
\hline Mild to moderate & $9(2.6)$ & $9(15.8)$ & 0 & \\
\hline Moderate & $11(3.2)$ & $11(19.3)$ & 0 & \\
\hline Moderate to severe & $1(0.3)$ & $1(1.8)$ & 0 & \\
\hline Severe & $2(0.6)$ & $2(3.5)$ & 0 & \\
\hline $\mathrm{AI}>$ mild & $23(6.8)$ & $23(40.4)$ & 0 & $<.001 *$ \\
\hline $\mathrm{MR} \geq$ moderate & $191(56.2)$ & $37(64.9)$ & $154(54.4)$ & .15 \\
\hline $\mathrm{TR} \geq$ moderate & $96(28.2)$ & $20(38.5)$ & $76(26.9)$ & .21 \\
\hline LVESD (mm) & $63.0 \pm 12.0$ & $59.4 \pm 10.2$ & $63.7 \pm 12.3$ & .10 \\
\hline LVEDD (mm) & $69.2 \pm 11.3$ & $69.0 \pm 11.0$ & $69.3 \pm 11.4$ & .85 \\
\hline Root diameter $(\mathrm{cm})$ & $3.21 \pm 0.45$ & $3.40 \pm 0.41$ & $3.16 \pm 0.43$ & $.002^{*}$ \\
\hline
\end{tabular}

\section{Predictors for Aortic Insufficiency Progression}

The clinical parameters before device implantation were analyzed to identify predictors for significant AI progression in each group. Among patients without

TABLE 3. Intraoperative data

\begin{tabular}{|c|c|c|c|c|}
\hline Variable & All & With repair & $\begin{array}{l}\text { Without } \\
\text { repair }\end{array}$ & $\begin{array}{c}P \\
\text { value }\end{array}$ \\
\hline Implanted device & & & & $.045^{*}$ \\
\hline HeartMate II & $281(82.6)$ & $55(96.5)$ & $226(79.9)$ & \\
\hline HeartWare & $36(10.6)$ & $1(1.8)$ & 35 (12.4) & \\
\hline Ventrassist & $9(2.6)$ & 0 & $9(3.2)$ & \\
\hline DuraHeart & $8(2.4)$ & $1(1.8)$ & $7(2.5)$ & \\
\hline DeBakey VAD & $6(1.8)$ & 0 & $6(2.1)$ & \\
\hline CPB time (min) & $94.4 \pm 48.1$ & $112.3 \pm 44.2$ & $90.7 \pm 48.1$ & $.002 *$ \\
\hline Crossclamp time (min) & $5.2 \pm 11.7$ & $23.3 \pm 9.9$ & $1.6 \pm 8.1$ & $<.001 *$ \\
\hline \multicolumn{5}{|l|}{ Transfusions (units) } \\
\hline Packed red blood cell & $1.6 \pm 2.5$ & $2.5 \pm 2.8$ & $1.4 \pm 2.4$ & $.009 *$ \\
\hline Fresh frozen plasma & $2.9 \pm 2.7$ & $3.8 \pm 2.9$ & $2.7 \pm 2.6$ & $.006^{*}$ \\
\hline Platelet & $9.4 \pm 7.4$ & $11.1 \pm 6.5$ & $9.0 \pm 7.5$ & $.051^{*}$ \\
\hline $\begin{array}{l}\text { Vasoactive-inotropic } \\
\text { score } \dagger\end{array}$ & $22.9 \pm 22.1$ & $26.5 \pm 13.3$ & $22.1 \pm 13.7$ & $.044^{*}$ \\
\hline \multicolumn{5}{|l|}{ Concomitant procedures } \\
\hline Mitral repair & $53(15.6)$ & $14(24.6)$ & $39(13.8)$ & $.041^{*}$ \\
\hline Ring annuloplasty & $45(12.9)$ & $12(21.1)$ & $33(11.7)$ & $.056^{*}$ \\
\hline Alfieri stitch & $8(2.4)$ & $2(3.5)$ & $6(2.1)$ & .57 \\
\hline Mitral replacement & $1(0.3)$ & 0 & $1(0.4)$ & .83 \\
\hline Tricuspid repair & $64(18.8)$ & $9(15.8)$ & $55(19.4)$ & .57 \\
\hline $\begin{array}{l}\text { Tricuspid } \\
\text { replacement }\end{array}$ & $11(3.2)$ & $3(5.3)$ & $8(2.8)$ & .42 \\
\hline RVAD implantation & $15(4.4)$ & $2(3.5)$ & $13(4.6)$ & .72 \\
\hline Centrimag & $13(4.0)$ & $2(3.6)$ & $11(4.0)$ & .89 \\
\hline HeartWare & $2(0.6)$ & 0 & $2(0.7)$ & .69 \\
\hline Device removal & $32(9.8)$ & 7 (12.7) & $25(9.1)$ & .42 \\
\hline Centrimag & $27(8.3)$ & $6(10.9)$ & $21(7.7)$ & .43 \\
\hline ECMO & $3(0.9)$ & $1(1.8)$ & $2(0.7)$ & .44 \\
\hline Impella & $2(0.6)$ & 0 & $2(0.7)$ & .69 \\
\hline Other & $29(8.5)$ & $4(7.3)$ & $25(8.8)$ & .65 \\
\hline
\end{tabular}

Values are $\mathrm{n}(\%)$, or mean $\pm \mathrm{SD}$, unless otherwise indicated. Centrimag is from Thoratec Corporation (Pleasanton, Calif); Impella is from ABIOMED, Inc (Danvers, Mass). VAD, Ventricular assist device; $C P B$, cardiopulmonary bypass; $R V A D$, right ventricular assist device; $E C M O$, extracorporeal membrane oxygenation. ${ }^{*} P<.05$. $\dagger$ Vasoactive-inotropic score (with doses in $\mu \mathrm{g} / \mathrm{kg} / \mathrm{min}$, unless otherwise indicated) $=$ dopamine dose + dobutamine dose $+100 \times$ epinephrine dose $+10 \times$ milrinone dose $+10,000 \times$ vasopressin dose $(\mathrm{U} / \mathrm{kg} / \mathrm{min})+100 \times$ norepinephrine dose. ${ }^{19}$ 
TABLE 4. Early and late postimplantation follow-up data on early mortality and adverse events

\begin{tabular}{|c|c|c|c|c|}
\hline Variable & All & With repair & $\begin{array}{l}\text { Without } \\
\text { repair }\end{array}$ & $\begin{array}{c}P \\
\text { value }\end{array}$ \\
\hline 30-d mortality & $16(4.7)$ & $2(3.5)$ & $14(4.9)$ & .64 \\
\hline In-hospital mortality & $30(8.8)$ & $7(12.3)$ & $23(8.1)$ & .31 \\
\hline ICU stay (d) & $10.4 \pm 10.6$ & $11.1 \pm 11.7$ & $10.2 \pm 10.4$ & .59 \\
\hline Hospital stay (d) & $45.7 \pm 36.3$ & $46.6 \pm 19.7$ & $45.6 \pm 38.3$ & .91 \\
\hline $\begin{array}{l}\text { Early postdevice/ } \\
\text { predischarge AI }\end{array}$ & & & & $<.001$ \\
\hline None & $135(39.7)$ & $39(68.4)$ & $96(33.9)$ & \\
\hline Trace & $152(44.7)$ & $16(28.1)$ & $136(48.1)$ & \\
\hline Mild & $36(10.6)$ & $1(1.8)$ & $35(12.4)$ & \\
\hline Mild to moderate & $6(1.8)$ & $1(1.8)$ & $5(1.8)$ & \\
\hline Moderate & $1(0.3)$ & 0 & $1(0.4)$ & \\
\hline N/A & $10(2.9)$ & 0 & $10(3.5)$ & \\
\hline \multicolumn{5}{|c|}{ Postoperative complications } \\
\hline Acute kidney injury & $56(16.5)$ & $10(17.5)$ & $46(16.3)$ & .72 \\
\hline CVVHD & $34(10.0)$ & $7(12.3)$ & $27(9.5)$ & .81 \\
\hline $\begin{array}{l}\text { Bleeding requiring } \\
\text { re-exploration }\end{array}$ & $56(16.5)$ & $8(14.0)$ & $48(17.0)$ & .59 \\
\hline CVA & $23(6.8)$ & $4(7.0)$ & $19(6.7)$ & .93 \\
\hline Urinary tract infection & $39(11.5)$ & $6(10.5)$ & $33(11.7)$ & .81 \\
\hline Sepsis & $27(7.9)$ & $4(7.0)$ & $23(8.1)$ & .78 \\
\hline $\begin{array}{l}\text { Atrial fibrillation/ } \\
\text { flutter }\end{array}$ & $79(23.2)$ & $10(17.6)$ & $69(24.4)$ & .27 \\
\hline $\mathrm{VT} / \mathrm{VF}$ & $80(23.5)$ & 14 (24.6) & $66(23.3)$ & .84 \\
\hline
\end{tabular}

Values are $\mathrm{n}(\%)$, or mean $\pm \mathrm{SD}$, unless otherwise indicated. $I C U$, Intensive care unit; $A I$, aortic insufficiency; N/A, not available; $C V V H D$, continuous veno-venous hemodialysis; $C V A$, cerebrovascular accident; $V T$, ventricular tachycardia; $V F$, ventricular fibrillation. $* P<.05$.

CAVC, factors that were multivariably significant were female gender and older age (hazard ratio, with $95 \%$ confidence interval: $2.80,1.45-5.41 ; P=.002$; and 1.54 , 1.22-1.93, respectively; $P<.001$ ) (Table E1). Conversely, no association was found between preimplantation clinical variables and AI progression among patients who had
CAVC. All 6 female patients in group A were free from significant AI progression.

\section{DISCUSSION}

We have demonstrated our outcomes of concurrent CAVC from a 10-year series of CF-LVAD experience. Our patient population has a high proportion of bridge-to-transplant patients among transplant recipients, with our current rate approaching $80 \%$. Significant waiting times, with the resultant prolonged device support duration, are becoming more common, applying currently to a majority of patients. Given these circumstances, we have been aggressive about performing concomitant CAVC.

The primary findings of interest in this study were: (1) the survival between groups after device implantation was similar; (2) despite significant pre-existing AI, the prevalence of significant $\mathrm{AI}$ after device implantation, in patients who received CAVC, was comparable to that in those without pre-existing AI or repair; (3) the efficacy and durability of repair seem more evident among DT patients; (4) repaired patients in the entire cohort and the DT cohort demonstrated a $57 \%$ and $69 \%$ decrease in the odds of significant AI progression, respectively, after adjusting for time effect and pre-existing AI degree.

With the improved durability of CF-LVAD, and the undersupply of donor hearts, attention has been focused on AI progression as a potential obstacle to device support, especially for those who require an extended waiting time for transplantation, or for DT patients. ${ }^{6}$ The mechanism involved in AI progression is likely multifactorial and may be related to failure of the AV opening and prolonged coaptation time, in addition to exposure of valvular tissue to persistently elevated retrograde pressure, resulting in structural changes such as commissural fusion of $\mathrm{AV}$ leaflets. ${ }^{4,6,20,21}$ Consequently, development of $\mathrm{AI}$

TABLE 5. Early and late postimplantation follow-up data, for all adverse events

\begin{tabular}{|c|c|c|c|c|c|}
\hline Adverse event & $\begin{array}{l}\text { With repair patients } \\
\quad(\mathbf{n}=\mathbf{5 7})\end{array}$ & Events rate* & $\begin{array}{l}\text { Without repair patients } \\
\qquad(\mathrm{n}=\mathbf{2 8 3})\end{array}$ & Events rate $\dagger$ & $P$ value \\
\hline Bleeding & $25(43.9)$ & $45(0.65)$ & $89(31.4)$ & $157(0.47)$ & .12 \\
\hline CVA & $8(14.0)$ & $10(0.14)$ & $43(15.2)$ & $46(0.14)$ & .88 \\
\hline \multicolumn{6}{|l|}{ Infection } \\
\hline Device-related & $6(10.5)$ & $18(0.26)$ & $32(11.3)$ & $70(0.21)$ & .45 \\
\hline Non-device-related & $16(28.1)$ & $23(0.33)$ & $36(12.7)$ & $42(0.12)$ & $.001 \ddagger$ \\
\hline Device malfunction & $3(5.3)$ & $5(0.072)$ & $49(17.3)$ & $58(0.17)$ & .065 \\
\hline Ventricular arrhythmias & $5(8.8)$ & $24(0.35)$ & $28(10.4)$ & $106(0.31)$ & .72 \\
\hline Pump thrombosis & $3(5.3)$ & $4(0.058)$ & $26(9.2)$ & $33(0.098)$ & .33 \\
\hline Pump replacement & $3(5.3)$ & $3(0.043)$ & $24(8.5)$ & $30(0.089)$ & .24 \\
\hline Due to thrombus & $3(5.3)$ & $3(0.043)$ & $10(3.5)$ & $14(0.042)$ & .95 \\
\hline Heart failure & $17(29.8)$ & $23(0.33)$ & $76(27.6)$ & $102(0.30)$ & .73 \\
\hline Right heart failure & $16(28.1)$ & $22(0.32)$ & $64(25.1)$ & $90(0.27)$ & .53 \\
\hline RVAD & $6(10.5)$ & $6(0.086)$ & $24(8.5)$ & $24(0.071)$ & .68 \\
\hline
\end{tabular}

Values are $\mathrm{n}(\%)$, unless otherwise indicated. CVA, Cerebrovascular accident; $R V A D$, right ventricular assist device. ${ }^{*}$ Events per 69.5 patient-years. $\dagger$ Events per 337.7 patient-years. $\ddagger P<.05$. 

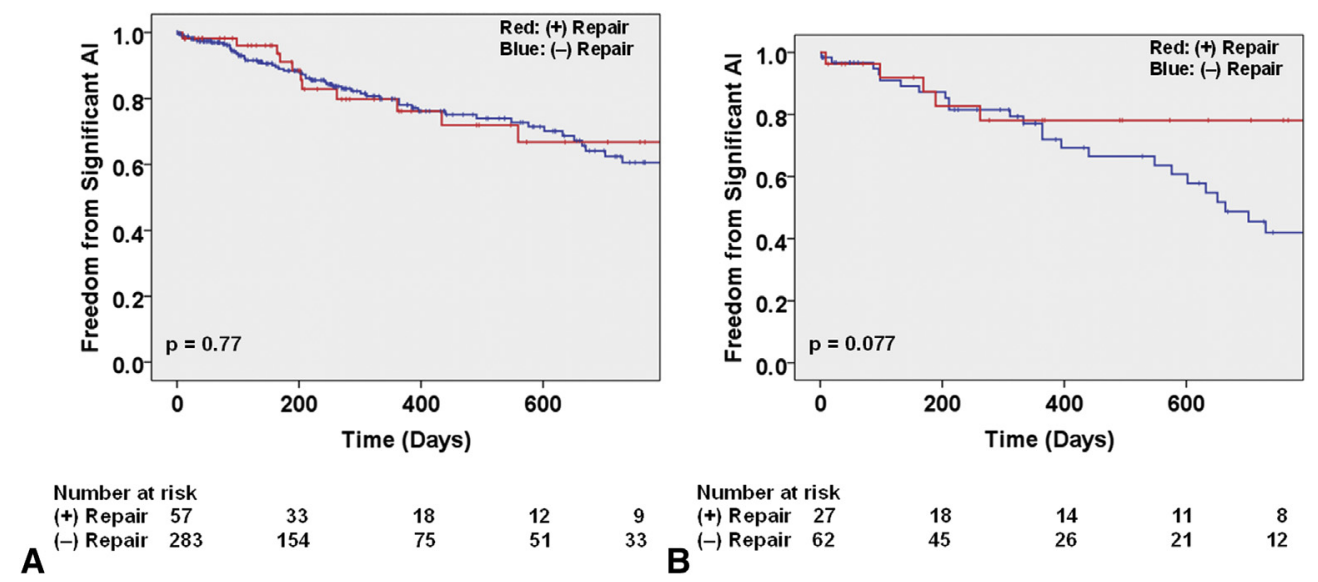

Number at risk

+) Repair 27

B

$\begin{array}{lllr}18 & 14 & 11 & 8 \\ 45 & 26 & 21 & 12\end{array}$

FIGURE 1. Freedom from > mild AI at 2 years after device implantation: comparison between patients with, versus without, aortic valve repair. A, Entire cohort; (B) Destination therapy subgroup. AI, Aortic insufficiency.

compromises device flow and can lead to ineffective device output and end-organ malperfusion because of the recycling of regurgitant blood. ${ }^{5}$ Several recent studies have suggested that greater aortic root diameter, higher pump speeds, older age, being female, and greater body surface area are potential risk factors associated with AI progression. 5,6,20,22

In contrast, our results demonstrated that female gender and older age are significant predictors of AI progression. Greater baseline aortic root diameter and body surface area were not predictive, although the former was significantly larger among those who had undergone AV repair; the latter was univariably significant (Tables 2 and E1). Although this hypothesis may be controversial, one possibility is that female and elderly patients are more susceptible to an increased growth rate or geometric changes of the aortic root, owing to CF-LVAD outflow, as both factors have been shown to be risk factors for thoracic aortic aneurysmal disease. ${ }^{23,24}$

By contrast, identification of clinical predictors for CAVC failure and resultant AI progression was difficult in such a small group $(n=57)$. However, 6 of $57(10.5 \%)$

TABLE 6. Longitudinal analysis using generalized mixed-effects model to assess the association between the treatment (repair vs no repair) and significant $\mathrm{AI}$ development during device support

\begin{tabular}{lrrr}
\hline \multicolumn{1}{c}{ Variable } & Estimate (SE) & OR (95\% CI) & $P$ value \\
\hline Entire cohort & & & \\
$\quad$ Repair vs no repair & $-0.84(0.39)$ & $0.43(0.20-0.93)$ & .032 \\
Time* & $0.63(0.07)$ & $1.88(1.64-2.16)$ & $<.001$ \\
Baseline AI degree $\dagger$ & $0.29(0.11)$ & $1.34(1.06-1.70)$ & .014 \\
Destination therapy cohort & & & \\
$\quad$ Repair vs no repair & $-1.18(0.47)$ & $0.31(0.12-0.79)$ & .015 \\
Time* & $0.58(0.09)$ & $1.79(1.48-2.18)$ & $<.001$ \\
Baseline AI degree $\dagger$ & $0.32(0.14)$ & $1.39(1.04-1.85)$ & .026 \\
\hline
\end{tabular}

All $P<.05 . S E$, Standard error; $O R$, odds ratio; $C I$, confidence interval; $A I$, aortic insufficiency. ${ }^{*}$ Time was fitted on the $\log$ scale. $\dagger$ Baseline AI was fitted using the original ordinal scale. patients in group A were women, and none of them developed significant AI during the follow-up period. Given the increased risk of significant AI, concurrent surgical correction may be particularly warranted in female and elderly patients.

Studies of the natural history of AI during device support have indicated that AI progression has a small clinical effect without any notable influence on survival. ${ }^{4,25}$ In fact, none of the CF-LVAD studies report an increase in mortality due to AI progression, ${ }^{25}$ because these patients can be surgically salvaged at the expense of added significant morbidity, as shown in our study (Table 7). Clinically relevant AI that is refractory to medical management occurred in 10 group B patients $(3.5 \%)$, which is $30 \%$ of those who developed $\geq$ moderate AI in our series. Atkins and colleagues ${ }^{26}$ reported reoperation using several techniques overall, on a total of 6 patients, for severe AI after implantation of an LVAD,. The incidence of reoperation in their cohort was $2.7 \%$, which was comparable to that in our series. Aortic insufficiency progression is not a "benign" complication of device support.

The question of what constitutes optimal surgical management of native AI is controversial. Moreover, the degree of AI that warrants concurrent surgical correction remains unclear. ${ }^{22}$ The recent guidelines ${ }^{27}$ recommend surgical intervention for $>$ mild AI, although this approach is based on past observations made on a limited number of patients who received varied $\mathrm{AV}$ procedures with relatively short-term device support. As for the efficacy and the durability of the CAVC technique, anecdotal experiences have been reported in a few studies. McKellar and colleagues ${ }^{13}$ reported on 18 patients who received concomitant CAVC, with a mean period of 441 days with excellent outcomes, although detailed follow-up data were not provided in their study. Schechter and colleagues ${ }^{12}$ described the same technique with promising mid-term 
TABLE 7. Early and late postimplantation follow-up data on surgical interventions performed for clinically significant AI

\begin{tabular}{|c|c|c|c|c|}
\hline Variable & All & With repair & Without repair & $P$ value \\
\hline Moderate or severe AI, n (\%) & $32(9.1)$ & $5(8.8)$ & $27(9.5)$ & .86 \\
\hline Aortic valve non-opener, n (\%) & $28(87.5)$ & $3(60.0)$ & $25(92.6)$ & .11 \\
\hline No. of patients who received surgical reintervention, $n(\%)$ & $11(3.2)$ & $1(1.8)$ & $10(3.5)$ & .49 \\
\hline No. of surgical reinterventions performed & 12 & 1 & 11 & \\
\hline Aortic valve repair & 5 & 0 & 5 & \\
\hline Aortic valve replacement & 1 & 0 & 1 & \\
\hline Aortic valve closure & 1 & 0 & 1 & \\
\hline AMPLATZER occluder & 1 & 0 & 1 & \\
\hline Heart transplant & 4 & 1 & 3 & \\
\hline Time to the surgical interventions undertaken (d) & $367 \pm 397$ (range: $21-1205)$ & 205 & $418 \pm 425$ & .65 \\
\hline
\end{tabular}

AI, Aortic insufficiency.

outcomes on 19 patients, of which 6 were not performed concomitantly.

More recently, Robertson and colleagues ${ }^{28}$ described various concomitant AV procedures, including 95 patients who had undergone AV repair. These data were collected from the Interagency Registry for Mechanically Assisted Circulatory Support (INTERMACS) dataset, although the type of repair was uncertain in $15.8 \%$ of cases. Moderate or greater AI after device implantation was observed in $18 \%$ of repaired patients by 6 to 12 months, which is a higher failure rate, compared with the studies mentioned earlier that have reported $0 \%$ to $10.5 \%$ recurrence rates of significant AI at a mean follow-up time of $>1$ year. ${ }^{12,13}$ However, use of a large registry limited access to potentially relevant clinical detail.

In contrast, the present study has a large sample size, with patients who have native AI treated consistently with the same technique. We excluded patients who had previously undergone AV surgery or received other AV procedures, to maintain the uniformity of the CAVC cohort. The prevalence of significant AI among those with CAVC was comparable to that among those without pre-existing $\mathrm{AI}$ or repair, despite the fact that many CAVC patients had significant pre-existing AI, which in some was even of $>$ moderate degree.

Further, longitudinal data analysis showed that CAVC is protective against AI progression after adjustment for varying device-support duration and baseline AI degree in each individual (Table 6). Additional CVACs were performed in 5 patients who developed a clinically significant de novo AI, refractory to medical management. Except for 1 patient who died from sepsis, the patients have been free of significant AI at latest follow-up, suggesting that CAVC can be utilized as a salvage procedure for clinically significant de novo AI.

The efficacy and durability of CAVC for addressing pre-device AI and preventing de novo AI were more clearly illustrated among DT patients (Figure 1, B; Table 6). The risk of AI development is cumulative over time. ${ }^{6,29} \mathrm{We}$ postulate that this observed tendency among DT patients might be derived mainly from the fact that DT patients are on device support for a much longer period than are bridge-to-transplant candidates (725 vs 322 days). Most previous reports are based on trials for bridge-totransplant patients, and few reported a follow-up time of $>1$ year on device support. Unlike bridge-to-transplant patients, most of whom bridged to transplant within 1 year, the DT cohort had fewer censoring events.

Another explanation of this superior efficacy includes the fact that the DT patients were a much older subgroup, with a mean age of 67 years (vs 53 years among the bridge-totransplant patients). As described, age was a significant predictor for significant AI progression among patients without CAVC, with a hazard ratio of 1.54 per 10 -year increment (Table E1). Considering the linear pattern of AI progression, more DT patients who have not undergone repair surgery may develop significant $\mathrm{AI}$ and require reoperation. Surgical reintervention in DT patients is not a trivial undertaking. Our results from this subgroup analysis indicate that the DT population in particular is the largest group who may potentially benefit from this technique.

Although 1 of advantages of this technique is its procedural simplicity, CAVC is not without risk. The cardiopulmonary bypass time in group A was significantly greater, with more-frequent usage of blood products and vasoactive drugs than for patients without repair. Nevertheless, our study did not show any survival difference between groups, despite the significant difference in these baseline and perioperative risk profiles. Furthermore, device exchange for pump thrombosis in the presence of CAVC was feasible and safe.

Finally, this repair technique may be applied to even those patients who have $\geq$ moderate AI. Thirteen of 14 patients $(93 \%)$ with pre-existing $\geq$ moderate AI have been free from recurrent $\mathrm{AI}$ at latest follow-up. By contrast, applicability of the technique to mild AI remains unclear. However, based on our findings, consideration of CAVC as a prophylactic repair in DT, elderly, and female patients may be appropriate, even if they have mild-degree native AI. 


\section{Limitations}

Our investigation is limited by its retrospective nature. Although this is the largest series describing the CAVC technique in the literature, the sample size still limits the statistical interpretation of the results. The serial follow-up echocardiographic studies, which often can be affected by hemodynamics, were performed in a variety of clinical settings, such as the intensive care unit and outpatient clinic. Additionally, the present data do not permit the comparison of CAVC with other surgical strategies, which remains an area for future investigation.

In conclusion, use of the CAVC technique at the time of CF-LVAD implantation is simple, effective, and durable in preventing AI progression, with a follow-up period that extends to 2 years after device implantation. In addition, clinically significant de novo AI that is refractory to medical management can be salvaged with this repair technique. This study provided data to support the practice of giving consideration to concurrent CAVC in DT or bridge-to-transplant patients who may need prolonged CF-LVAD support.

\section{Conflict of Interest Statement}

Y.N. has been a consultant for the Thoratec Corporation. All other authors have nothing to disclose with regard to commercial support.

\section{References}

1. Pagani FD, Miller LW, Russell SD, Aaronson KD, John R, Boyle AJ, et al. Extended mechanical circulatory support with a continuous-flow rotary left ventricular assist device. J Am Coll Cardiol. 2009;54:312-21.

2. Kirklin JK, Naftel DC, Kormos RL, Stevenson LW, Pagani FD, Miller MA, et al. Fifth INTERMACS annual report: Risk factor analysis from more than 6,000 mechanical circulatory support patients. J Heart Lung Transplant. 2013;32: 141-56.

3. Starling RC, Moazami N, Silvestry SC, Ewald G, Rogers JG, Milano CA, et al. Unexpected abrupt increase in left ventricular assist device thrombosis. $N$ Engl J Med. 2014;370:33-40.

4. Rajagopal K, Daneshmand MA, Patel CB, Ganapathi AM, Schechter MA, Rogers JG, et al. Natural history and clinical effect of aortic valve regurgitation after left ventricular assist device implantation. J Thorac Cardiovasc Surg. 2013; 145:1373-9.

5. Cowger J, Pagani FD, Haft JW, Romano MA, Aaronson KD, Kolias TJ. The development of aortic insufficiency in left ventricular assist device-supported patients. Circ Heart Fail. 2010;3:668-74.

6. Jorde UP, Uriel N, Nahumi N, Bejar D, Gonzalez-Costello J, Thomas SS, et al. Prevalence, significance, and management of aortic insufficiency in continuous flow left ventricular assist device recipients. Circ Heart Fail. 2014; 7:310-9.

7. Adamson RM, Dembitsky WP, Baradarian S, Chammas J, May-Newman K, Chillcott S, et al. Aortic valve closure associated with HeartMate left ventricular device support: technical considerations and long-term results. J Heart Lung Transplant. 2011;30:576-82.

8. Cohn WE, Demirozu ZT, Frazier OH. Surgical closure of left ventricular outflow tract after left ventricular assist device implantation in patients with aortic valve pathology. J Heart Lung Transplant. 2011;30:59-63.

9. Feldman CM, Silver MA, Sobieski MA, Slaughter MS. Management of aortic insufficiency with continuous flow left ventricular assist devices: bioprosthetic valve replacement. J Heart Lung Transplant. 2006;25:1410-2.

10. Bietry R, Balsam LB, Saric M, McElhinney DB, Katz S, Deanda A Jr, et al. Percutaneous intervention for recurrent aortic insufficiency in a patient with a left ventricular assist device and a centrally oversewn aortic valve. Circ Heart Fail. 2013;6:e43-4.

11. Park SJ, Liao KK, Segurola R, Madhu KP, Miller LW. Management of aortic insufficiency in patients with left ventricular assist devices: a simple coaptation stitch method (Park's stitch). J Thorac Cardiovasc Surg. 2004;127:264-6.

12. Schechter MA, Joseph JT, Krishnamoorthy A, Emanuel Finet J, Ganapathi AM, Lodge AJ, et al. Efficacy and durability of central oversewing for treatment of aortic insufficiency in patients with continuous-flow left ventricular assist devices. J Heart Lung Transplant. 2014;33:937-42.

13. McKellar SH, Deo S, Daly RC, Durham LA III, Joyce LD, Stulak JM, et al Durability of central aortic valve closure in patients with continuous flow left ventricular assist devices. J Thorac Cardiovasc Surg. 2014;147:344-8.

14. Uriel N, Morrison KA, Garan AR, Kato TS, Yuzefpolskaya M, Latif F, et al. Development of a novel echocardiography ramp test for speed optimization and diagnosis of device thrombosis in continuous-flow left ventricular assist devices: the Columbia ramp study. J Am Coll Cardiol. 2012:60:1764-75.

15. Zoghbi WA, Enriquez-Sarano M, Foster E, Grayburn PA, Kraft CD, Levine RA, et al. Recommendations for evaluation of the severity of native valvular regurgitation with two-dimensional and Doppler echocardiography. J Am Soc Echocardiogr. 2003;16:777-802.

16. Takayama H, Yang JA, Naka Y. Tips on tuning each device: technical pearls Cardiol Clin. 2011:29:551-6.

17. Ware JH. Linear models for longitudinal continuous data. In: Fitzmaurice GM, Laird NM, Ware JH, eds. Applied Longitudinal Analysis. 2nd ed. Hoboken, NJ: Wiley; 2011:95.

18. Son MK, Chang SA, Kwak JH, Lim HJ, Park SJ, Choi JO, et al. Comparative measurement of aortic root by transthoracic echocardiography in normal Korean population based on two different guidelines. Cardiovasc Ultrasound. 2013;11:28.

19. Nguyen HV, Havalad V, Aponte-Patel L, Murata AY, Wang DY, Rusanov A, et al Temporary biventricular pacing decreases the vasoactive-inotropic score after cardiac surgery: a substudy of a randomized clinical trial. J Thorac Cardiovasc Surg. 2013;146:296-301.

20. Pak SW, Uriel N, Takayama H, Cappleman S, Song R, Colombo PC, et al. Prevalence of de novo aortic insufficiency during long-term support with left ventricular assist devices. J Heart Lung Transplant. 2010;29:1172-6.

21. Mudd JO, Cuda JD, Halushka M, Soderlund KA, Conte JV, Russell SD. Fusion of aortic valve commissures in patients supported by a continuous axial flow left ventricular assist device. J Heart Lung Transplant. 2008;27:1269-74.

22. John R, Naka Y, Park SJ, Sai-Sudhakar C, Salerno C, Sundareswaran KS, et al Impact of concurrent surgical valve procedures in patients receiving continuous-flow devices. J Thorac Cardiovasc Surg. 2014;147:581-9.

23. Rylski B, Desjardins B, Moser W, Bavaria JE, Milewski RK. Gender-related changes in aortic geometry throughout life. Eur J Cardiothorac Surg. 2014;45: 805-11.

24. Coady MA, Rizzo J, Goldstein LJ, Elefteriades JA. Natural history, pathogenesis, and etiology of thoracic aortic aneurysms and dissections. Cardiol Clin. 1999;17: 615-35.

25. Cowger JA, Aaronson KD, Romano MA, Haft J, Pagani FD. Consequences of aortic insufficiency during long-term axial continuous-flow left ventricular assist device support. J Heart Lung Transplant. 2014;33:1233-40.

26. Atkins BZ, Hashmi ZA, Ganapathi AM, Harrison JK, Hughes GC, Rogers JG et al. Surgical correction of aortic valve insufficiency after left ventricular assist device implantation. J Thorac Cardiovasc Surg. 2013;146:1247-52.

27. Feldman D, Pamboukian SV, Teuteberg JJ, Birks E, Lietz K, Moore SA, et al. The 2013 International Society for Heart and Lung Transplantation guidelines for mechanical circulatory support: executive summary. J Heart Lung Transplant. 2013;32:157-87.

28. Robertson JO, Naftel DC, Myers SL, Prasad S, Mertz GD, Itoh A, et al. Concomitant aortic valve procedures in patients undergoing implantation of continuous-flow left ventricular assist devices: an INTERMACS database analysis. J Heart Lung Transplant. 2015;34:797-805.

29. Cowger J, Rao V, Massey T, Sun B, May-Newman K, Jorde U, et al Comprehensive review and suggested strategies for the detection and management of aortic insufficiency in patients with a continuous-flow left ventricular assist device. J Heart Lung Transplant. 2015;34:149-57.

Key Words: mechanical circulatory assistance, transplantation—heart, valve disease, aortic valve, repair 


\section{Discussion}

Dr R. Davis (Durham, NC). Congratulations on an excellent presentation. It is interesting that one quarter of the patients are failing at 1 year, and in the overall cohort, it is approximately one third that fail at a later time point, and there seems to be a difference between your DT group and your overall group with respect to these failures. I will first just ask: What do you think that difference is? Why do you think your overall group did worse than the DT subgroup?

Dr S. Fukuhara. First, as I briefly mentioned, the DT cohort patients obviously stayed on device support much longer than those in the bridge-to-transplant cohort. Second, they have many fewer censoring events because they do not get transplanted. Another thing is that-but I did not include the data on these slides-1 of the predictors for AI progression, based on our analysis, was older age. The DT patients were older than the bridge-to-transplant patients. That was probably another reason that the repair was protective in the DT cohort.

Dr Davis. I would think that would be just the oppositethat the longer the on-device duration, the greater the likelihood of AI. Did some of those that you were planning on being DT receive transplantation because of the degree of AI?

Dr Fukuhara. Your question is about someone in the DT cohort who got a heart transplant?

Dr Davis. Well, you said in the overall cohort it was approximately one third of the patients who actually have a failure or a progression of AI in the overall cohort, but when you break out just your DT patients, it was approximately $20 \%$ to $22 \%$. So that finding goes somewhat against the idea that longer duration would be more likely to be associated with AI, because theoretically your bridge-totransplant group should have had shorter on-device times.

Dr Fukuhara. I see. As I showed in the Kaplan-Meier curve, patients who did not get a concurrent repair in the DT cohort obviously had progressed to de novo AI; on the other hand, patients who got repaired basically stayed at the same degree of AI, despite the presence of a significantly worse degree of baseline AI. That is what we observed based on our analysis.

Dr Davis. What I really want to get at is: What are the predictors that indicate a patient is going to progress with AI? In your article, you really did not find anything within the group in which you did the repairs. But you were just using echo assessments of your aortic valve size and aortic size. I do not know if you actually looked at more CTs, or what would be more-sensitive impressions of the aortic size and the aortic valve as potential predictors of development of AI.

The other question is: What were you doing, both in terms of the flow rates and pump speed, as well as drug therapy for AI protection? Was there a difference between the groups that might explain that?

Dr Y. Naka (New York, NY). I can answer your question. In terms of the etiology - or how we can predict either AI or the kind of patient who will develop more AI-some studies from our group suggest that if we maintain normal aortic valve function, such as open and closed, with enough volume inside the left ventricle, we might prevent AI progression, because other studies show that AI is associated with aortic valve fusion peripherally, in the central opening of the aortic valve.

Another issue is the DT. Very few patients develop clinically meaningful AI, which required some aortic valve intervention or going to transplant based on transplant eligibility, but fewer patients in the AI repair group went to urgent transplant or something similar to it.

Dr Davis. So what are you doing - are you trying now to have lower flow and more ejection?

Dr Naka. Well, yes. So we tend to decrease the flow compared with our historical early experience. We used to use a HeartMate II, in particular, that went up to $>9000 \mathrm{rpm}$. Now, our de novo HeartMate II goes up to $>8600$ to $9000 \mathrm{rpm}$. 


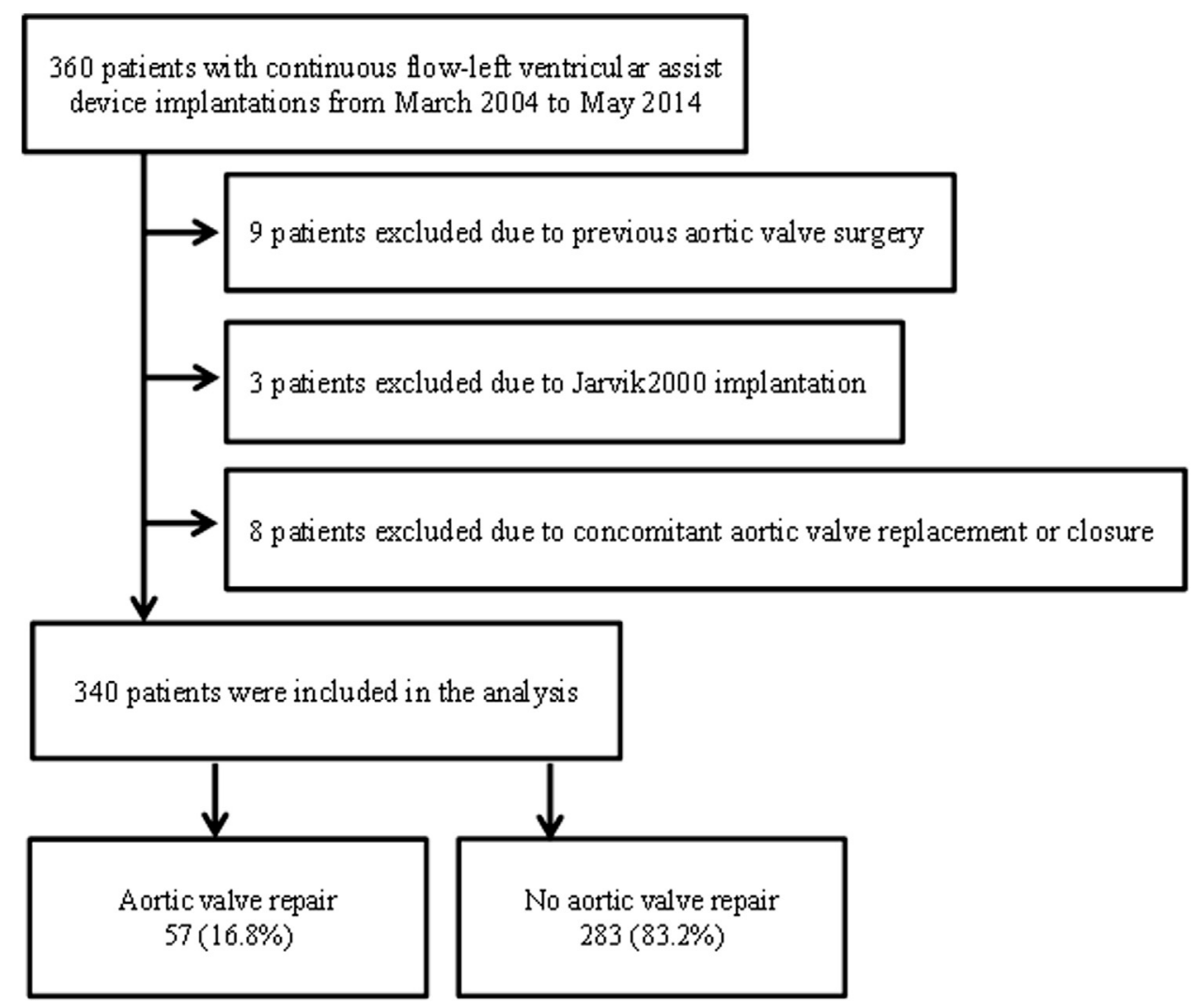

FIGURE E1. Cohort derivation diagram.

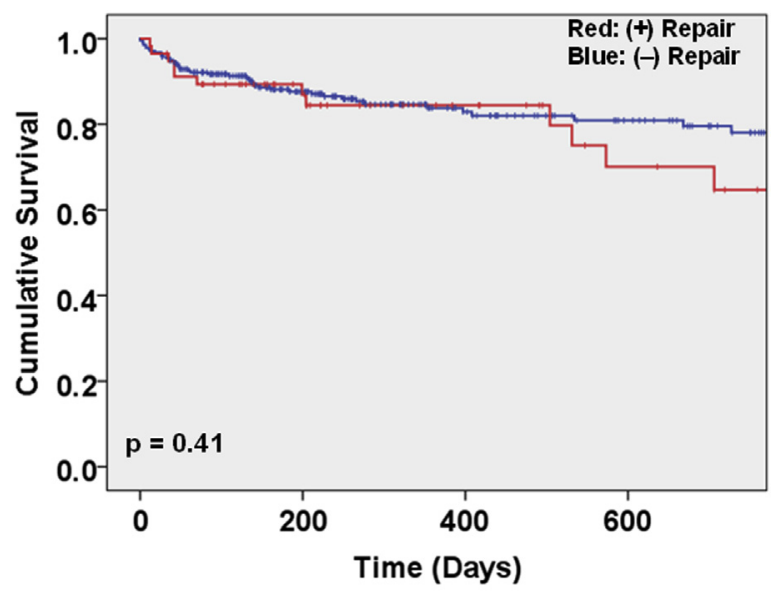

Number at risk

(+) Repair 57

(-) Repair 283

37

23

1413

FIGURE E2. Kaplan-Meier survival curve for on-device survival at

2 years after device implantation, for patients with, versus without, aortic valve repair. 
TABLE E1. Predictors for progression of significant AI among patients without aortic valve repair

\begin{tabular}{lccccc}
\hline & \multicolumn{2}{c}{ Univariable } & & \multicolumn{2}{c}{ Multivariable } \\
\cline { 2 - 3 } \cline { 5 - 6 } \multicolumn{1}{c}{ Variable } & HR $(\mathbf{9 5} \% \mathbf{C I})$ & $\boldsymbol{P}$ value & & HR $(\mathbf{9 5} \% \mathbf{C I})$ & $\boldsymbol{P}$ value \\
\hline BSA & $0.30(0.10-0.88)$ & .028 & & $1.01(0.30-3.40)$ & .99 \\
Gender, female & $2.11(1.22-3.64)$ & .007 & & $2.80(1.45-5.41)$ & $.002 *$ \\
Age $\dagger$ & $1.44(1.18-1.77)$ & $<.001$ & & $1.54(1.22-1.93)$ & $<.001 *$ \\
Baseline AI & & & & & \\
$\quad$ None & Reference & & & Reference & \\
$\quad$ Trace & $1.01(0.58-1.76)$ & .97 & & $1.12(0.64-1.97)$ & .69 \\
$\quad$ Mild & $2.09(0.64-6.85)$ & .22 & & $2.04(0.60-6.86)$ & .25 \\
\hline
\end{tabular}

$H R$, Hazard ratio; $C I$, confidence interval; $B S A$, body surface area; $A I$, aortic insufficiency. $* P<.05$. $†$ The HR for age is shown for a 10 -year increment.
TABLE E3. Each time point for Kaplan-Meier survival curve, for freedom from $>$ mild AI at 2 years after device implantation

\begin{tabular}{cccc}
\hline & With repair & & Without repair \\
\cline { 2 - 3 } Time (d) & $\begin{array}{c}\text { Freedom from significant AI } \\
(\mathbf{9 5} \% \mathbf{C I})\end{array}$ & $\begin{array}{c}\text { Freedom from significant AI } \\
(\mathbf{9 5} \% \mathbf{C I})\end{array}$ \\
\hline Entire cohort & & \\
200 & $88.5(83.6-93.4)$ & & $87.9(85.7-90.1)$ \\
400 & $76.2(69.1-83.4)$ & & $76.2(72.8-79.6)$ \\
600 & $66.8(57.9-75.7)$ & & $70.1(66.0-74.2)$ \\
730 & $66.8(57.9-75.7)$ & & $60.6(55.5-65.7)$ \\
Destination therapy subgroup & & \\
200 & $82.7(74.8-90.6)$ & & $85.3(80.5-90.1)$ \\
400 & $78.1(69.4-86.8)$ & & $69.3(62.5-76.1)$ \\
600 & $78.1(69.4-86.8)$ & & $57.9(50.2-65.6)$ \\
730 & $78.1(69.4-86.8)$ & $42.0(33.7-50.3)$ \\
\hline
\end{tabular}

See Figure $1, A$ and $B . A I$, Aortic insufficiency; $C I$, confidence interval.

TABLE E2. Kaplan-Meier curve time points, for on-device survival at 2 years after device implantation

\begin{tabular}{lcc}
\hline Time (d) & $\begin{array}{c}\text { With repair } \\
\text { Survival }(\mathbf{9 5} \% \mathbf{C I})\end{array}$ & $\begin{array}{c}\text { Without repair } \\
\text { Survival }(\mathbf{9 5} \% \mathbf{C I})\end{array}$ \\
\hline 200 & $87.1(82.5-91.7)$ & $87.8(85.8-90.2)$ \\
400 & $84.7(79.6-89.8)$ & $83.1(80.5-85.7)$ \\
600 & $70.7(62.2-79.2)$ & $80.0(76.9-83.1)$ \\
730 & $65.7(56.4-75.0)$ & $78.6(75.3-81.9)$ \\
\hline
\end{tabular}

See Figure E2 for survival curve. $C I$, Confidence interval. 